\title{
Estrutura da comunidade de liquens corticícolas em área urbana e rural no município de Novo Hamburgo, Rio Grande do Sul, Brasil
}

\author{
Paula Graziela Lehnen', Márcia Isabel Käffer², Fabiane Lucheta² \& Jairo Lizandro Schmitt¹,2 \\ 'Universidade Feevale, Laboratório de Botânica. Rodovia RS 239, 2755, bairro Vila Nova, CEP 93352-075, Novo Hamburgo, RS, Brasil. \\ paulalehnen@feevale.br \\ ${ }^{2}$ Universidade Feevale, Programa de Pós-Graduação em Qualidade Ambiental. Rodovia RS 239, 2755, bairro Vila Nova, CEP 93352-075, \\ Novo Hamburgo, RS, Brasil.mkaffer9617@gmail.com.br, fabiane.lucheta@gmail.com; jairols@feevale.br
}

Recebido em 05.IX.2016

Aceito em 27.IV.2017

DOI: $10.21826 / 2446-8231201772107$

\begin{abstract}
RESUMO - O objetivo do estudo foi analisar a composição e estrutura da comunidade de liquens foliosos e fruticosos corticícolas em área urbana e rural do município de Novo Hamburgo, RS. Foram analisados dez forófitos em cada área e o mapeamento das espécies realizado pelo método do acetato. Foram analisados os parâmetros: riqueza, frequência, cobertura e valor de importância em cada área. No total foram identificadas 55 espécies para as duas áreas. A riqueza foi similar entre as áreas: urbana (33) e rural (32). As espécies mais frequentes foram Dirinaria picta (Sw.) Clem. \& Shear e Parmotrema reticulatum (Nyl.) Hale, enquanto Canoparmelia texana (Tuck.) Elix \& Hale e Parmotrema tinctorum (Nyl.) Hale obtiveram os maiores valores de importância. A área rural apresentou melhores condições para o desenvolvimento dos liquens. Este trabalho contribui para o conhecimento da micobiota na região, podendo ser utilizado em futuros estudos de monitoramento ambiental.
\end{abstract}

Palavras-chave: diversidade, foliosos, fruticosos, fungos liquenizados

ABSTRACT - Community structure of corticolous lichens in urban and rural areas in the municipality of Novo Hamburgo, Rio Grande do Sul, Brazil. In this paper we analyzed the composition and the corticolous lichen community structure of foliose and fruticose species in urban and rural areas in the municipality of Novo Hamburgo, RS. We analyzed ten phorophytes in each area and mapped species by using the acetate method. The parameters analyzed in each area were: richness, frequency, coverage and importance value. A total of 55 lichen species were identified for both areas. Richness was similar among areas: urban (33) and rural (32). The most common species were Dirinaria picta (Sw.) Clem. \& Shear and Parmotrema reticulatum (Nyl.) Hale, while Canoparmelia texana (Tuck.) Elix \& Hale and Parmotrema tinctorum (Nyl.) Hale, had the highest importance values. The rural area had better conditions for lichen development. This work contributes to the knowledge of the mycobiota in the region and may be used for future studies on environmental monitoring.

Keywords: diversity, foliose, fruticose, lichenized fungi

\section{INTRODUÇÃO}

Os liquens são organismos compostos pela associação de um fungo (micobionte) e algas verdes e/ou cianobactérias (fotobionte) resultando num corpo vegetativo denominado talo (Nash III 2008). Pesquisas recentes demonstraram a presença do fungo Cyphobasidium (Basidomycete) como um terceiro componente nesta associação (Spribille et al. 2016). Estes organismos possuem vida longa e são encontrados nos mais variados substratos e ambientes, podendo se fixar em troncos e ramificações, no solo, sobre rochas ou folhas (Brodo et al. 2001). Algumas características refletem a versatilidade destes organismos, como em resistir a variações ambientais, podendo sobreviver a grandes períodos de seca e sendo capazes de absorver vapor de água do ar suficiente para manter uma atividade metabólica basal (Maccheroni et al. 2010).

Os fatores ambientais podem influenciar na distribuição destes organismos ao longo e ao redor dos troncos, principalmente a luz e a umidade (Marcelli 1992, Ellis 2012). As características físicas e químicas da casca dos forófitos como textura, dureza, retenção de água, $\mathrm{pH}$ e composição de macro e micronutrientes são de fundamental importância para o estabelecimento das espécies (Brodo 1973, Marcelli 1996, Seaward 2008).

Desde o século XIX os liquens são utilizados como indicadores ambientais, originando diversas pesquisas visando o monitoramento ambiental, seja por meio de estudos para avaliar a qualidade do ar em ambientes urbano/ industriais (Le Blanc \& De Sloover 1970, Martins et al. 2008, Käffer et al. 2012) ou em áreas florestais (Hauck et al. 2013, Käffer \& Martins 2014, Käffer et al. 2015, 2016a, b).

Atualmente, estima-se entre 13.500 e 20.000 espécies de liquens em todo o mundo (Lücking et al. 2009, 2017). Para o Brasil, Marcelli (2004) listou 287 gêneros e 2.874 espécies. Destas, 912 espécies estão listadas para o estado do Rio Grande do Sul (Spielmann 2006). No entanto, para o Brasil ainda são poucos os estudos sobre a estrutura da comunidade de liquens corticícolas (Marcelli 1992, Käffer et al. 2009, 2010, 2011, 2016 a,b, Cáceres et al. 2007, 2008, Fleig \& Grüninger 2008, Martins \& Marcelli 2011, Koch et al. 2012, 2013, Lucheta \& Martins 2014). E, para o município de Novo Hamburgo, RS, até o momento não há registros publicados de trabalhos sobre a comunidade de 
liquens. Desta forma, este estudo tem por objetivo avaliar a composição e a estrutura da comunidade de liquens corticícolas foliosos e fruticosos, em área urbana e rural no município de Novo Hamburgo, RS.

\section{MATERIAL E MÉTODOS}

\section{Área de estudo}

O município de Novo Hamburgo possui uma área de $223,821 \mathrm{~km}^{2}$ com densidade demográfica de aproximadamente $1,07 \mathrm{hab} / \mathrm{km}^{2}$. Está localizado cerca de $40 \mathrm{~km}$ da capital do Estado do Rio Grande do Sul, fazendo parte da Região Metropolitana de Porto Alegre e integrando a Bacia Hidrográfica do Rio dos Sinos (IBGE 2016). A área rural de Novo Hamburgo possui extensão territorial de 156 $\mathrm{km}^{2}$, equivalente a dois terços do território do município. A mesma encontra-se dividida em microbairros caracterizados por propriedades rurais, áreas de cultivo e pastagem. Possui também áreas de proteção ambiental (APP) abrangendo o banhado do Rio dos Sinos, considerado um ecossistema importante para a preservação da fauna e da flora da região (Novo Hamburgo 2015). De acordo com a classificação climática de Köppen-Geiger (Peel et al. 2007), o clima é do tipo Cfa, com temperatura média de $19,4^{\circ} \mathrm{C}$ e pluviosidade média anual de $1467 \mathrm{~mm}$ (Climate 2016). A vegetação da região pertence à fisionomia da Floresta Estacional Semidecidual (Teixeira et al. 1986).

\section{Seleção das áreas de amostragem}

Foram selecionadas duas áreas distintas para a amostragem da comunidade de liquens corticícolas no município de Novo Hamburgo: uma na zona urbana (área I) e outra na zona rural (área II). As duas áreas estão a uma distância de aproximadamente $22 \mathrm{~km}$ uma da outra (Fig. 1).

A área I localiza-se no Campus II da Universidade Feevale, junto à rodovia ERS-239, e possui uma área de 15 ha $\left(29^{\circ} 39^{\prime} 88.5^{\prime \prime}\right.$ 'S e $\left.51^{\circ} 07^{\prime} 15.0^{\prime \prime} \mathrm{W}\right)$ situada a 90 metros de altitude (Universidade Feevale 2015). A área II está localizada em uma propriedade particular (Sítio São Luiz), bairro Lomba Grande, na localidade de Morro dos Bois $\left(29^{\circ} 47^{\prime} 80.4^{\prime \prime} \mathrm{S}\right.$ e $\left.51^{\circ} 00^{\prime} 30.2^{\prime \prime} \mathrm{W}\right)$ situada a 55 metros de altitude. Esta área possui cerca de 40 ha distribuídos entre morros, matas (áreas e Mata Atlântica), campos e riachos (Sítio São Luiz 2015).

\section{Mapeamento dos liquens}

Em cada área de amostragem foram selecionados 10 forófitos dispostos isoladamente, com circunferência a altura do peito (CAP) acima de $20 \mathrm{~cm}$, com fuste reto, sem ramificações abaixo de $150 \mathrm{~cm}$, com estrutura da casca semelhantes (Tab. 1). Na área I (urbana) os forófitos se encontravam em área de estacionamento e/ou canteiros, enquanto que na área II (rural) em áreas ajardinadas do sítio.

Para o mapeamento dos liquens corticícolas dos grupos foliosos e fruticosos foi empregado o método das folhas

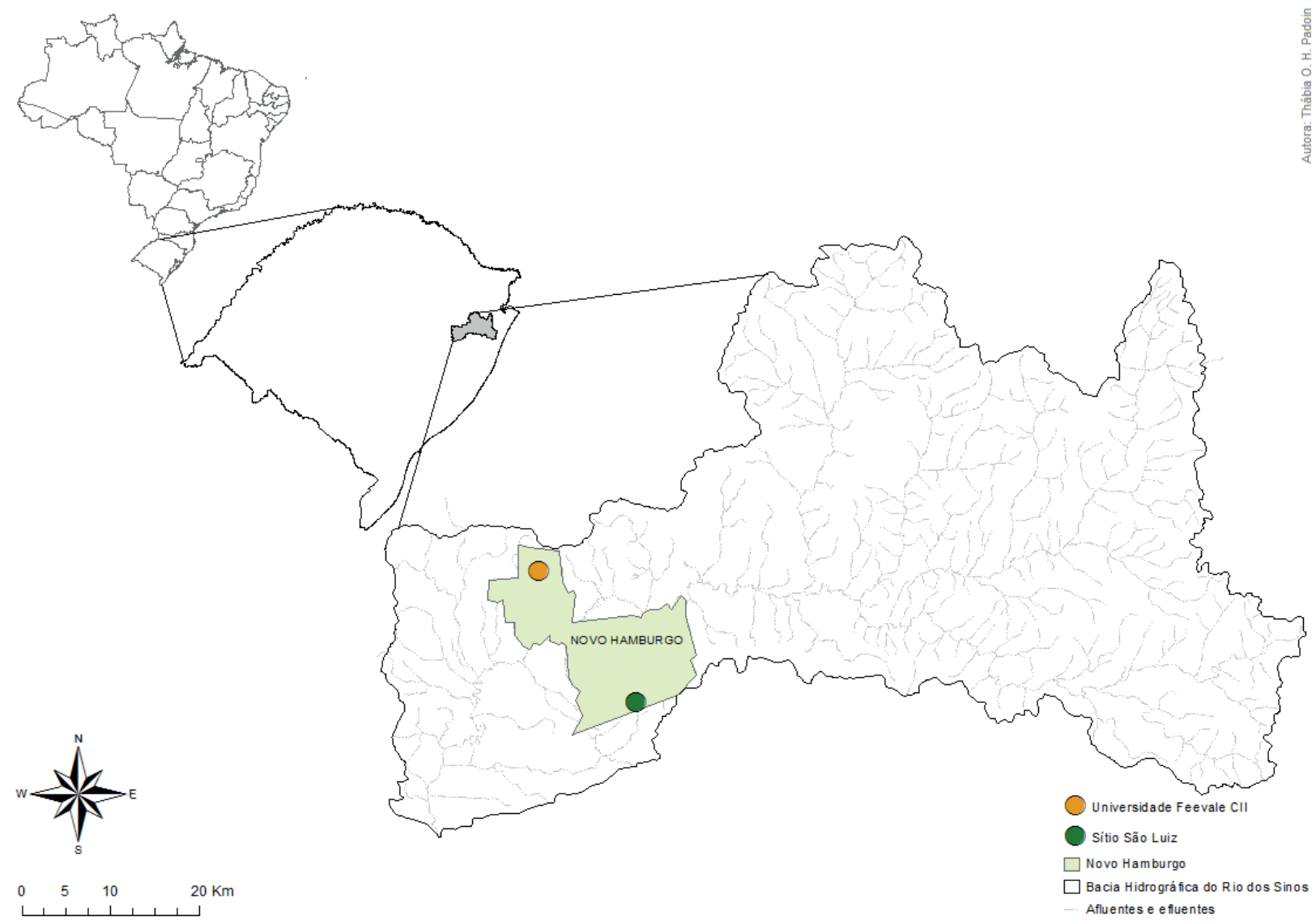

Fig.1. Mapa de localização das áreas de estudo em Novo Hamburgo, RS, Brasil. 
Tabela 1. Espécies de forófitos analisados nas áreas I (urbana) e II (rural) para o mapeamento dos liquens, com seu respectivo intervalo de circunferência a altura do peito. Legenda: $\mathrm{NI}$ = número de indivíduos, $\mathrm{CAP}$ = circunferência a altura do peito.

\begin{tabular}{|c|c|c|c|c|c|}
\hline \multirow{2}{*}{ Espécie } & \multirow{2}{*}{ Nome popular } & \multirow{2}{*}{ NI } & \multicolumn{2}{|c|}{$\mathrm{CAP}(\mathrm{cm})$} & \multirow[t]{2}{*}{$\begin{array}{c}\text { Estrutura } \\
\text { Casca }\end{array}$} \\
\hline & & & Área I & Área II & \\
\hline Caesalpinia pluviosa DC. & Sibipiruna & 1 & 55 & & Áspera \\
\hline Cinnamoтит verum J. Presl & Canela & 1 & 60 & & Fissurada \\
\hline Handroanthus heptaphylla (Vell.) Mattos & Ipê roxo & 4 & $41-76$ & 82 & Fissurada \\
\hline Hovenia dulcis Thunb. & Uva do Japão & 3 & & $72-85$ & Fissurada \\
\hline Jacaranda mimosifolia D. Don & Jacaranda & 3 & $70-84$ & & Fissurada \\
\hline Melia azedarach $\mathrm{L}$. & Cinamomo & 2 & & $108-108,5$ & Fissurada \\
\hline Morus nigra L. & Amoreira & 2 & & $67,5-161,5$ & Fissurada \\
\hline Peltophorum dubium (Spreng.) Taub. & Canafístula & 1 & 54 & & Fissurada \\
\hline Salix humboldtiana Willd & Salseiro & 2 & & $124-264$ & Fissurada \\
\hline Tipuana tipu (Benth.) Kuntze & Tipuana & 1 & 72 & & Fissurada \\
\hline
\end{tabular}

de acetato (Käffer et al. 2015), utilizando três folhas de acetato de $20 \mathrm{~cm} \times 20 \mathrm{~cm}$, dispostas ao longo do tronco dos forófitos. A amostragem foi realizada a partir de $80 \mathrm{~cm}$ acima do solo até $140 \mathrm{~cm}$, totalizando uma área de $120 \mathrm{~cm}^{2}$, na face em que os liquens se encontravam mais abundantes. Posteriormente, foi realizado o delineamento do talo das amostras foliosas e fruticosas, com a finalidade de realizar o cálculo da cobertura total de cada espécie, em laboratório.

\section{Identificação das espécies}

As amostras liquênicas demarcadas que não puderam ser identificados em campo foram coletadas e armazenadas para identificação no Laboratório de Botânica, da Universidade Feevale. Para a realização de análises morfológicas e químicas dessas amostras foi utilizado microscópio estereoscópico e óptico e para as análises químicas foram realizados testes de coloração no córtex e medula, com os reagentes hidróxido de potássio $20 \%(\mathrm{KOH})$, hipoclorito de sódio comercial 1\% ( $\mathrm{NaClO})$ e parafenilenodiamina $(\mathrm{P})$. Realizou-se também teste de fluorescência por exposição à luz ultravioleta (UV) em medula e córtex.

A identificação taxonômica das espécies foi feita com o uso de bibliografia especializada comumente empregada em taxonomia de liquens e auxílio de alguns especialistas. O material foi herborizado e inserido na Coleção Botânica do Curso de Ciências Biológicas da Universidade Feevale, Novo Hamburgo, RS. Posteriormente, as amostras mais representativas de cada área serão enviadas ao Herbarium Anchieta (PACA), em São Leopoldo, RS para catalogação.

\section{Análise dos dados}

A riqueza foi considerada como o número total de espécies de liquens ocorrentes nos dez forófitos, em cada área analisada. A frequência foi baseada na presença/ausência das espécies em cada forófito. Para a estimativa da cobertura dos liquens foi utilizada a soma total da cobertura de todos os talos, para cada folha de acetato e em todos os forófitos analisados. O valor de importância (VI) para cada espécie foi calculado somando-se os valores de frequência relativa (FR) e cobertura relativa (CR) para cada área amostrada.
Os dados de riqueza e cobertura de liquens por forófito foram comparados entre as duas áreas (urbana e rural) pelo teste de Mann-Whitney a 5\% de probabilidade. Esta análise foi realizada no Programa estatístico Paleontological Statistics Software Package for Education and Data Analysis (PAST), versão 3.01 (Hammer et al. 2001).

\section{RESULTADOS}

\section{Composição da comunidade}

Foram identificadas 55 espécies de liquens, distribuídas em sete famílias e 16 gêneros (Tab. 2), sendo $78,2 \%$ de talo folioso e $21,8 \%$ de talo fruticoso. Destas espécies, $94,5 \%$ estão associadas à clorofícea e somente $5,5 \%$ a cianobactéria como fotobionte.

A família mais representativa nas duas áreas foi Parmeliaceae com 36 espécies, seguida de Physciaceae com oito, Collemataceae e Lobariaceae, ambas com três espécies, Ramalinaceae e Caliciaceae com dois e Teloschistaceae um representante (Fig. 2).

\section{Estrutura da comunidade}

$\mathrm{Na}$ área I (urbana) foram encontradas 33 espécies, sendo Parmeliaceae a família mais rica com 23 espécies, seguida de Physciaceae com seis, Caliciaceae com duas e Collemataceae e Ramalinaceae ambas com uma espécie. A espécie liquênica com maior frequência foi Dirinaria picta (Sw.) Clem. \& Shear ocorrendo em 80\% dos forófitos, seguida de Canoparmelia texana (Tuck.) Elix \& Hale presente em $60 \%$ dos forófitos e, Parmotrema austrosinense (Zahlbr.) Hale, Parmotrema tinctorum (Nyl.) Hale, Parmotrema reticulatum (Nyl.) Hale e Crespoa carneopruinata (Zahlbr.) Lendemer \& B.P. Hodk ocorrendo em $50 \%$ dos forófitos amostrados (Tab. 3). Em relação à cobertura de liquens na área urbana, Canoparmelia texana destacou-se com 19,41\% de cobertura, seguida de P. tinctorum com $11,15 \%$ e D. picta com $6,42 \%$ (Tab. $3)$. Quanto ao valor de importância, as espécies mais representativas foram Canoparmelia texana com 26,91\% do VI total $\left(\sum=200\right)$, seguida de Parmotrema tinctorum com $17,41 \%$ e Dirinaria picta com $16,43 \%$ (Tab. 3 ). 
Tabela 2. Composição da comunidade liquênica corticícola distribuída por família, área de ocorrência e grupo morfológico. Legenda: Área I= Urbana, Área II = Rural, Folioso=Fol, Fruticoso=Fru

\begin{tabular}{|c|c|c|c|c|}
\hline Família & Espécie & Área I & Área II & Grupo morfológico \\
\hline \multirow[t]{2}{*}{ Caliciaceae } & Dirinaria confluens (Fries) D.D. Awasthi & $\mathrm{x}$ & & Fol \\
\hline & Dirinaria picta (Sw.) Clem. \& Shear & $\mathrm{x}$ & $\mathrm{x}$ & Fol \\
\hline \multirow[t]{3}{*}{ Collemataceae } & Collema fasciculare Sommerf. & & $\mathrm{x}$ & Fol \\
\hline & Leptogium austroamericanum (Malme) C.W. Dodge & $\mathrm{x}$ & & Fol \\
\hline & Leptogium sp. 1 & & $\mathrm{x}$ & Fol \\
\hline \multirow[t]{3}{*}{ Lobariaceae } & Crocodia aurata (Ach.) Link & & $\mathrm{x}$ & Fol \\
\hline & Crocodia clathrata (De Not.) Trevis. & & $\mathrm{x}$ & Fol \\
\hline & Ricasolia erosa (Eschw.) Nyl. & & $\mathrm{x}$ & Fol \\
\hline \multirow[t]{36}{*}{ Parmeliaceae } & Canoparmela texana (Tuck.) Elix \& Hale & $\mathrm{x}$ & $\mathrm{x}$ & Fol \\
\hline & Crespoa carneopruinata (Zahlbr.) Lendemer \& B.P. Hodk. & $\mathrm{x}$ & & Fol \\
\hline & Hypotrachyna livida (Taylor) Hale & $\mathrm{x}$ & $\mathrm{x}$ & Fol \\
\hline & Hypotrachyna polydactyla (Krog \& Swinscow) Nash & & $\mathrm{x}$ & Fol \\
\hline & Hypotrachyna sp. 1 & $\mathrm{x}$ & & Fol \\
\hline & Parmotrema austrosinense (Zahlbr.) Hale & $\mathrm{x}$ & & Fol \\
\hline & Parmotrema cetratum (Zahlbr.) Hale & & $\mathrm{x}$ & Fol \\
\hline & Parmotrema consors (Nyl.) Elix \& Hale & $\mathrm{x}$ & & Fol \\
\hline & Parmotrema eciliatum (Nyl.) Hale & $\mathrm{x}$ & & Fol \\
\hline & Parmotrema lindmanii (Lynge) Elix \& Hale & $\mathrm{x}$ & & Fol \\
\hline & Parmotrema melanothrix (Mont.) Hale & $\mathrm{x}$ & $\mathrm{x}$ & Fol \\
\hline & Parmotrema praesorediosum (Nyl.) Hale & $\mathrm{x}$ & & Fol \\
\hline & Parmotrema rampoddense (Nyl.) Hale & & $\mathrm{x}$ & Fol \\
\hline & Parmotrema recipiendum (Nyl.) Hale & $\mathrm{x}$ & & Fol \\
\hline & Parmotrema reticulatum (Taylor) M. Choisy & $\mathrm{x}$ & $\mathrm{x}$ & Fol \\
\hline & Parmotrema sancti-angeli (Lynge) Hale & & $\mathrm{x}$ & Fol \\
\hline & Parmotrema simulans (Hale) Hale & $\mathrm{x}$ & & Fol \\
\hline & Parmotrema subrugatum (Kremp.) Hale & $\mathrm{x}$ & & Fol \\
\hline & Parmotrema subsumptum (Nyl.) Hale & $\mathrm{x}$ & & Fol \\
\hline & Parmotrema tinctorum (Nyl.) Hale & $\mathrm{x}$ & $\mathrm{x}$ & Fol \\
\hline & Punctelia canaliculata (Lynge) Krog & $\mathrm{x}$ & & Fol \\
\hline & Punctelia constantimontium Sérus. & & $\mathrm{x}$ & Fol \\
\hline & Punctelia cf. osorioi (Hale) Hale & $\mathrm{x}$ & & Fol \\
\hline & Punctelia riograndensis (Lynge) Krog & $\mathrm{x}$ & & Fol \\
\hline & Punctelia subrudecta (Nyl.) Krog & $\mathrm{x}$ & & Fol \\
\hline & Punctelia sp.1 & $\mathrm{x}$ & & Fol \\
\hline & Punctelia sp. 2 & & $\mathrm{x}$ & Fol \\
\hline & Usnea rubicunda Stirt. & $\mathrm{x}$ & $\mathrm{x}$ & Fru \\
\hline & Usnea sp.1 & & $\mathrm{x}$ & Fru \\
\hline & Usnea sp. 2 & & $\mathrm{x}$ & Fru \\
\hline & Usnea sp. 3 & & $\mathrm{x}$ & Fru \\
\hline & Usnea sp. 4 & & $\mathrm{x}$ & Fru \\
\hline & Usnea sp. 5 & & $\mathrm{x}$ & Fru \\
\hline & Usnea sp. 6 & & $\mathrm{x}$ & Fru \\
\hline & Usnea sp. 7 & $\mathrm{x}$ & $\mathrm{x}$ & Fru \\
\hline & Usnea sp. 8 & & $\mathrm{x}$ & Fru \\
\hline \multirow[t]{8}{*}{ Physciaceae } & Heterodermia leucomela (L.) Poelt & & $\mathrm{x}$ & Fol \\
\hline & Heterodermia obscurata (Nyl.) Trevis & $\mathrm{x}$ & $\mathrm{x}$ & Fol \\
\hline & Hyperphyscia cochlearis Scutari & $\mathrm{x}$ & & Fol \\
\hline & Physcia aipolia (Ehrenb. ex Humb.) Fürnrohr & $\mathrm{x}$ & & Fol \\
\hline & Physcia atrostriata Moberg & & $\mathrm{x}$ & Fol \\
\hline & Physcia krogiae Moberg & $\mathrm{x}$ & & Fol \\
\hline & Physcia sorediosa (Vain.) Lynge & $\mathrm{x}$ & & Fol \\
\hline & Physcia sp. 1 & $\mathrm{x}$ & & Fol \\
\hline \multirow[t]{2}{*}{ Ramalinaceae } & Ramalina celatri Ach & $\mathrm{x}$ & $\mathrm{x}$ & Fru \\
\hline & Ramalina peruviana Ach. & & $\mathrm{x}$ & Fru \\
\hline Teloschistaceae & Teloschistes exilis (Michx.) Vain. & & $\mathrm{x}$ & Fru \\
\hline
\end{tabular}




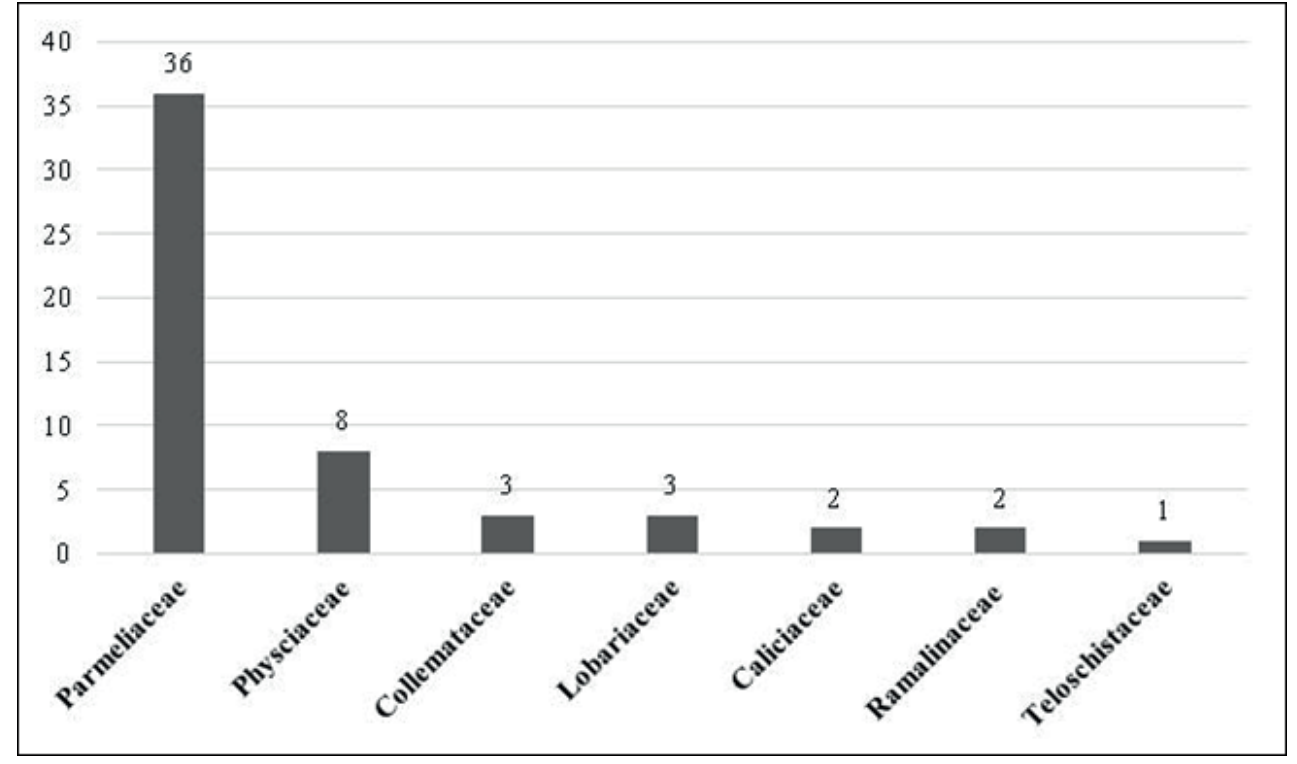

Fig. 2. Distribuição das espécies por família encontradas nas duas áreas de estudo.

Tabela 3. Parâmetros estruturais da comunidade liquênica na área $\mathrm{I}$ (urbana), Legenda: $\mathrm{CA}=$ cobertura absoluta, $\mathrm{CR}=$ cobertura relativa $(\%), \mathrm{FA}=$ frequência absoluta, $\mathrm{FR}=$ frequência relativa (\%) e VI= valor de Importância.

\begin{tabular}{|c|c|c|c|c|c|c|}
\hline Espécie & Riqueza & $\mathrm{CA}$ & $\mathrm{CR}$ & FA & FR & VI \\
\hline Canoparmelia texana & 6 & 88,80 & 19,41 & 0,60 & 7,50 & 26,91 \\
\hline Parmotrema tinctorum & 5 & 51,04 & 11,15 & 0,50 & 6,25 & 17,41 \\
\hline Dirinaria picta & 8 & 29,40 & 6,42 & 0,80 & 10,00 & 16,43 \\
\hline Parmotrema austrosinense & 5 & 26,69 & 5,83 & 0,50 & 6,25 & 12,08 \\
\hline Punctelia riograndensis & 2 & 42,76 & 9,34 & 0,20 & 2,50 & 11,85 \\
\hline Parmotrema reticulatum & 5 & 24,33 & 5,31 & 0,50 & 6,25 & 11,57 \\
\hline Crespoa carneopruinata & 5 & 23,60 & 5,15 & 0,50 & 6,25 & 11,41 \\
\hline Parmotrema lindmanii & 4 & 26,80 & 5,85 & 0,40 & 5,00 & 10,86 \\
\hline Physcia aipolia & 4 & 17,86 & 3,90 & 0,40 & 5,00 & 8,90 \\
\hline Dirinaria confluens & 4 & 13,67 & 2,98 & 0,40 & 5,00 & 7,99 \\
\hline Heterodermia obscurata & 2 & 25,03 & 5,47 & 0,20 & 2,50 & 7,97 \\
\hline Parmotrema simulans & 1 & 23,37 & 5,10 & 0,10 & 1,25 & 6,36 \\
\hline Ramalina celastri & 4 & 4,57 & 0,99 & 0,40 & 5,00 & 6,00 \\
\hline Punctelia sp. 1 & 1 & 12,20 & 2,66 & 0,10 & 1,25 & 3,92 \\
\hline Parmotrema recipiendum & 2 & 4,73 & 1,03 & 0,20 & 2,50 & 3,53 \\
\hline Punctelia subrudecta & 1 & 9,00 & 1,96 & 0,10 & 1,25 & 3,22 \\
\hline Hyperphyscia cochlearis & 2 & 2,80 & 0,61 & 0,20 & 2,50 & 3,11 \\
\hline Parmotrema eciliatum & 2 & 2,60 & 0,56 & 0,20 & 2,50 & 3,07 \\
\hline Punctelia canaliculata & 2 & 1,67 & 0,36 & 0,20 & 2,50 & 2,87 \\
\hline Parmotrema praesorediosum & 2 & 1,60 & 0,35 & 0,20 & 2,50 & 2,85 \\
\hline Usnea sp. 7 & 1 & 3,40 & 0,74 & 0,10 & 1,25 & 1,99 \\
\hline Parmotrema subsumptum & 1 & 3,27 & 0,71 & 0,10 & 1,25 & 1,96 \\
\hline Physcia krogiae & 1 & 2,76 & 0,60 & 0,10 & 1,25 & 1,85 \\
\hline Parmotrema melanothrix & 1 & 2,57 & 0,56 & 0,10 & 1,25 & 1,81 \\
\hline Physcia sorediosa & 1 & 2,07 & 0,45 & 0,10 & 1,25 & 1,70 \\
\hline Punctelia osorioi & 1 & 1,90 & 0,41 & 0,10 & 1,25 & 1,67 \\
\hline Usnea rubicunda & 1 & 1,80 & 0,39 & 0,10 & 1,25 & 1,64 \\
\hline Parmotrema subrugatum & 1 & 1,77 & 0,38 & 0,10 & 1,25 & 1,64 \\
\hline Parmotrema consors & 1 & 1,63 & 0,35 & 0,10 & 1,25 & 1,61 \\
\hline Hypotrachyna livida & 1 & 1,6 & 0,35 & 0,10 & 1,25 & 1,60 \\
\hline Leptogium austroamericanum & 1 & 1,17 & 0,25 & 0,10 & 1,25 & 1,51 \\
\hline Hypotrachyna sp. 1 & 1 & 0,80 & 0,17 & 0,10 & 1,25 & 1,42 \\
\hline \multirow[t]{2}{*}{ Physcia sp. 1} & 1 & 0,23 & 0,05 & 0,10 & 1,25 & 1,30 \\
\hline & & 457,49 & 100 & 8,00 & 100 & 200 \\
\hline
\end{tabular}


$\mathrm{Na}$ área II (rural) foram registradas 32 espécies, sendo que Parmeliaceae apresentou a maior riqueza, com 20 espécies, seguida de Physciaceae e Lobariaceae com três, Collemataceae e Ramalinaceae, ambas com duas espécies e Caliciaceae e Teloschistaceae ambas com uma espécie (Tab. 4).

A espécie com maior frequência na área rural foi Parmotrema reticulatum ocorrendo em $80 \%$ dos forófitos, seguida de Parmotrema tinctorum em $70 \%$ dos forófitos e Ramalina peruviana Ach em $60 \%$ dos forófitos amostrados. Quanto à cobertura das espécies, Parmotrema tinctorum representou 22,97\% de cobertura, seguida de Parmotrema reticulatum com 18,57\% e Punctelia sp. 2 com 12,83\% (Tab. 4). Para o valor de importância, pode-se observar que as espécies que apresentaram os maiores valores foram $P$. tinctorum com $32,31 \%$ do VI total $\left(\sum=200\right)$, seguida de P. reticulatum com 29,24\% e Punctelia sp. 2 com 15,50\% (Tab. 4).

$\mathrm{Na}$ análise da riqueza e cobertura de liquens nos forófitos, entre as áreas analisadas (urbana e rural) não foram constatadas diferenças significativas (Tab. 5).

Tabela 4. Parâmetros estruturais da comunidade liquênica na área II (rural). Legenda: $\mathrm{CA}=$ cobertura absoluta, $\mathrm{CR}=$ cobertura relativa (\%), $\mathrm{FA}=$ frequência absoluta, $\mathrm{FR}=$ frequência relativa (\%) e VI= valor de Importância.

\begin{tabular}{|c|c|c|c|c|c|c|}
\hline Espécie & Riqueza & $\mathrm{CA}$ & $\mathrm{CR}$ & FA & FR & VI \\
\hline Parmotrema tinctorum & 7 & 116,73 & 22,97 & 0,70 & 9,33 & 32,31 \\
\hline Parmotrema reticulatum & 8 & 94,35 & 18,57 & 0,80 & 10,67 & 29,24 \\
\hline Punctelia sp. 2 & 2 & 65,19 & 12,83 & 0,20 & 2,67 & 15,50 \\
\hline Heterodermia obscurata & 4 & 41,50 & 8,16 & 0,40 & 5,33 & 13,50 \\
\hline Parmotrema cetratum & 5 & 31,03 & 6,10 & 0,50 & 6,67 & 12,77 \\
\hline Ramalina peruviana & 6 & 12,45 & 2,45 & 0,60 & 8,00 & 10,45 \\
\hline Ricasolia erosa & 1 & 33,73 & 6,63 & 0,10 & 1,33 & 7,97 \\
\hline Collema fasciculare & 1 & 30,77 & 6,05 & 0,10 & 1,33 & 7,39 \\
\hline Punctelia constantimontium & 2 & 23,83 & 4,69 & 0,20 & 2,67 & 7,36 \\
\hline Canoparmelia texana & 4 & 4,36 & 0,85 & 0,40 & 5,33 & 6,19 \\
\hline Heterodermia leucomela & 1 & 17,83 & 3,51 & 0,10 & 1,33 & 4,84 \\
\hline Parmotrema melanothrix & 3 & 3,10 & 0,61 & 0,30 & 4,00 & 4,61 \\
\hline Ramalina celastri & 3 & 1,40 & 0,27 & 0,30 & 4,00 & 4,28 \\
\hline Dirinaria picta & 3 & 1,17 & 0,23 & 0,30 & 4,00 & 4,23 \\
\hline Usnea sp. 4 & 2 & 5,74 & 1,13 & 0,20 & 2,67 & 3,80 \\
\hline Parmotrema sancti-angeli & 2 & 4,87 & 0,95 & 0,20 & 2,67 & 3,63 \\
\hline Usnea sp. 2 & 2 & 3,3 & 0,65 & 0,20 & 2,67 & 3,32 \\
\hline Usnea sp. 7 & 2 & 2,93 & 0,57 & 0,20 & 2,67 & 3,24 \\
\hline Hypotrachyna livida & 2 & 1,83 & 0,36 & 0,20 & 2,67 & 3,03 \\
\hline Physcia atrostriata & 2 & 1,39 & 0,27 & 0,20 & 2,67 & 2,94 \\
\hline Teloschistes exilis & 2 & 0,53 & 0,10 & 0,20 & 2,67 & 2,77 \\
\hline Crocodia aurata & 1 & 2,30 & 0,45 & 0,10 & 1,33 & 1,79 \\
\hline Usnea rubicunda & 1 & 2,13 & 0,41 & 0,10 & 1,33 & 1,75 \\
\hline Usnea sp. 6 & 1 & 1,80 & 0,35 & 0,10 & 1,33 & 1,69 \\
\hline Usnea sp. 3 & 1 & 0,63 & 0,12 & 0,10 & 1,33 & 1,46 \\
\hline Leptogium sp. 1 & 1 & 0,57 & 0,11 & 0,10 & 1,33 & 1,45 \\
\hline Hypotrachyna polydactyla & 1 & 0,40 & 0,07 & 0,10 & 1,33 & 1,41 \\
\hline Usnea sp. 1 & 1 & 0,40 & 0,07 & 0,10 & 1,33 & 1,41 \\
\hline Crocodia clathrata & 1 & 0,37 & 0,07 & 0,10 & 1,33 & 1,41 \\
\hline Parmotrema rampoddense & 1 & 0,30 & 0,05 & 0,10 & 1,33 & 1,39 \\
\hline Usnea sp. 5 & 1 & 0,20 & 0,03 & 0,10 & 1,33 & 1,37 \\
\hline \multirow[t]{2}{*}{ Usnea sp. 8} & 1 & 0,90 & 0,17 & 0,10 & 1,33 & 1,51 \\
\hline & & 508,03 & 100 & 7,50 & 100 & 200 \\
\hline
\end{tabular}

Tabela 5. Dados de riqueza e cobertura de liquens, entre as áreas I (urbana) e II (rural).

\begin{tabular}{lcccc}
\hline Dados & Área & Média & Desvio padrão & $\mathrm{U}^{1}$ \\
\hline \multirow{2}{*}{ Riqueza } & I (Urbana) & 8,0 & 2,44 & 49,5 \\
& II (Rural) & 7,4 & 2,01 & 1,00 \\
Cobertura & I (Urbana) & & & \\
& II (Rural) & 47,43 & 9,03 & 46,0 \\
\hline
\end{tabular}

$\mathrm{U}^{1}$. Valor do teste de Mann-Whitney 


\section{DISCUSSÃO}

Das famílias mais representativas registradas nas áreas de estudo, Parmeliaceae é conhecida por comportar o maior número (2.765) de espécies liquênicas (Lücking et al. 2017), enquanto que Physciaceae juntamente com outras espécies do grupo morfológico crostoso são usualmente registradas em ambientes urbanos (Marcelli 1998). Além disso, Parmeliaceae apresenta um grande número de taxa, cerca de 2.400 espécies, distribuídas em 85 gêneros (Blanco et al. 2005). Estudos realizados no Estado corroboram com os resultados encontrados nas áreas analisadas, sendo que $65,45 \%$ das espécies registradas pertencem a esta família (Käffer \& Martins-Mazzitelli 2005, Martins \& Marcelli 2011, Koch et al. 2012, Lucheta \& Martins 2014, Käffer et al. 2015, 2016b).

Das espécies com maior frequência e cobertura na área I destacam-se Dirinaria picta, Canoparmelia texana e Parmotrema tinctorum. Estas espécies pertencem ao grupo morfológico folioso e fazem parte das famílias Caliciaceae e Parmeliaceae, respectivamente. As duas primeiras espécies apresentam talos pequenos se comparados com P. tinctorum, porém a maior ocorrência destas espécies pode estar relacionada com suas características morfológicas e ecológicas. A ocorrência de espécies liquênicas foliosas de lobos estreitos e/ou largos está associada às condições ambientais, como radiação solar e disponibilidade de água (Giordani et al. 2013). As duas primeiras espécies também apresentaram os maiores valores de importância para a comunidade na área I (urbana). Trabalhos realizados em áreas urbano-industriais na região metropolitana do Rio Grande do Sul com a comunidade liquênica reportam estas espécies como predominantes na estrutura da micobiota (Martins et al. 2008, Käffer et al. 2011, 2016b, Lucheta \& Martins 2014).

Para a área II (rural), as espécies Parmotrema reticulatum, Parmotrema tinctorum e Ramalina peruviana, esta última pertencente ao grupo morfológico fruticoso, apresentaram os maiores valores de frequência, cobertura e valor de importância na comunidade. A ocorrência destas espécies na área pode também estar relacionada por seus aspectos ecológicos, pois ocorrem em áreas abertas, assim como características morfológicas. Espécies do gênero Parmotrema se caracterizam por apresentar talos geralmente grandes (Spielmann \& Marcelli 2008). A espécie $R$. peruviana cresce em ramos e troncos de arbustos e árvores em áreas abertas (Kashiwadani \& Kalb 1993).

A predominância destas espécies em ambas as áreas também pode ter sido influenciada pela estrutura dos forófitos que apresentavam casca fissurada, além de estarem localizados em locais isolados. A estrutura da casca, como a rugosidade e porosidade, estão relacionadas com diferenças na estrutura da comunidade de liquens (Brodo 1973, Ellis 2012). Os troncos das árvores também podem ser considerados como unidades ecológicas para o estabelecimento das espécies (Lie et al. 2009).

No que se refere às espécies de talo fruticoso, a maior ocorrência foi na área II (rural), especialmente espécies do gênero Usnea. A presença de liquens fruticosos está associada a locais mais úmidos (Giordani et al. 2013, Käffer \& Martins 2014, Matos et al. 2015). Segundo MartinsMazzitelli et al. (2006), os liquens fruticosos são os mais sensíveis à poluição atmosférica, sendo os primeiros a desaparecerem em áreas muito degradadas, podendo-se sugerir que no ambiente rural amostrado as condições microclimáticas associado às características ambientais da região estavam mais favoráveis para a ocorrência destes espécimes quando comparado à área urbana analisada.

$\mathrm{O}$ registro de Ricasolia erosa (Eschw.) Nyl na área II (rural) se destaca neste estudo. Esta espécie é característica de ambientes mais preservados e úmidos (Käffer \& Martins 2014), o que confere uma melhor condição ambiental a esta área. Este gênero tem sido citado como indicador de ecossistemas florestais preservados, com alta continuidade ecológica (Campbell \& Fredeen 2004, Liira \& Sepp 2009, Gjerde et al. 2012). De outro lado o baixo índice de liquens que portam cianobactéria nas áreas I e II (5,5\%) pode estar atribuído ao fato de que estes organismos estarem normalmente relacionados a ambientes úmidos e sombreados (Sierk 1964, Giordani et al. 2013, Matos et al. 2015). Neste estudo houve apenas a ocorrência de uma espécie do gênero Collema e duas espécies do gênero Leptogium. Em áreas urbanas a ocorrência de espécies do gênero Leptogium está associada à sua adaptabilidade a diferentes tipos de ambientes (Wolseley 1991), onde os talos crescem geralmente nas partes mais úmidas de vários tipos de ambientes e o grau de especificidade apresentada em relação ao substrato, e que pode variar de acordo com a espécie (Swinscow \& Krog 1988).

Embora não tenhamos encontrado diferenças significativas nos dados de riqueza $(\mathrm{U}=49,5, p=1,00) \mathrm{e}$ cobertura $(\mathrm{U}=46,0, p=0,79)$ de liquens dentre as duas áreas analisadas verificamos que a distinção entre a área I (urbana) e II (rural) se deve em função da composição de espécies. Na área rural $40,62 \%$ das espécies pertencem aos liquens que portam cianobactéricas (Leptogium e Collema) ou são do grupo morfológico fruticoso (Ramalina, Usnea e Teloschistes), além dos gêneros Crocodia e Ricasolia que também não foram constatados na área urbana. Espécies liquênicas do tipo fruticoso, assim como aquelas associadas às cianobactérias são mais suscetíveis as alterações ambientais, especialmente pelas variações nos fatores climáticos, como temperatura, umidade e luminosidade ocorrentes em ambientes urbanos (Martins-Mazzitelli et al. 2006, Giordani et al. 2013, Matos et al. 2015).

Embora a riqueza de espécies obtida neste trabalho tenha sido inferior ao inventariado em outras pesquisas para a região metropolitana de Porto Alegre, este estudo vem a contribuir para o conhecimento da micota liquenizada corticícola para o trecho inferior da Bacia do Rio dos Sinos, no estado do Rio Grande do Sul. Este estudo também pode servir como subsídio para futuros trabalhos, incluindo monitoramento ambiental na região e para o acompanhamento de possíveis alterações do ambiente 
que possam vir a acontecer. Além disso, o registro de Ricasolia erosa, associada com espécies do gênero Usnea e Teloschistes que foram praticamente exclusivas na área rural, caracterizam este local como mais propício para o desenvolvimento e estabelecimento das espécies liquênicas que requerem melhores condições ambientais, principalmente nos aspectos microclimáticos.

\section{AGRADECIMENTOS}

Agradecemos à Universidade Feevale pela infraestrutura disponibilizada e ao Sr. Luiz Antônio Daudt, proprietário do Sítio São Luiz, pela disponibilização da área para estudo. A liquenóloga Dra. Patrícia Jungbluth, da Universidade Federal de Santa Maria, campi Palmeira das Missões pela confirmação de algumas espécies do gênero Physcia e a Mestranda Thábia O. H. Padoin, do Laboratório de Botânica da Universidade Feevale pela compilação do mapa da área de estudo.

\section{REFERÊNCIAS}

Blanco, O., Crespo, A., Divakar, P.K., Elix, J.A. \& Lumbsch, H.T. 2005. Molecular phylogeny of parmotremoid lichens (Ascomycota, Parmeliaceae). Mycologia 97(1): 150-159.

Brodo, I.M. 1973. Substratum ecology. In The Lichens (M.E. Hale, ed.). Academic Press, New York, p. 401-436.

Brodo, I.M., Sharnoff, S.D. \& Sharnoff, S. 2001. Lichens of North America. Yale University Press, New Haven. 795 p.

Cáceres, M.E., Lücking, R. \& Rambold, G. 2007. Phorophyte specifity and evironmetal parameters versus stochasticity as determinants for species composition of corticolous crustose lichen communities in the Atlantic rain forest of northeastern Brazil. Mycological Progress 6: 117-136.

Cáceres, M.E., Lücking, R. \& Rambold, G. 2008. Corticolous microlichens in northeastern Brazil: habitat differentiation between coastal Mata Atlântica, Caatinga and Brejos de Altitude. Bryologist 111: 98-117.

Campbell, J. \& Fredeen, A.L. 2004. Lobaria pulmonaria abundance as an indicator of macrolichen diversity in Interior Cedar-Hemlock forests of east-central British Columbia. Canadian Journal of Botany 82: 970-982.

Climate-Date.org 2016. Dados climáticos para cidades mundiais. Disponível em: <http://pt.climate-data.org/> Acessado em 31.05.2016.

Ellis, C.J. 2012. Lichen epiphyte diversity: A species, community and trait-based review. Perspectives in Plant Ecology, Evolution and Systematics 14: 131-152

Fleig, M. \& Grüninger, W. 2008. Liquens da Floresta com Araucária no Rio Grande do Sul. Pró-Mata: Guia de Campo 3. University of Tübingen, Germany, p. 1-217.

Gjerde, I., Blom, H.H., Lindblom, L., Sætersdal, M. \& Schei, F.H. 2012. Community assembly in epiphytic lichens in early stages of colonization. Ecology 93(4): 749-759.

Giordani, P., Incerti, G., Rizzi, G., Rellini, I., Nimis, P.L. \& Modenesi, P. 2013. Functional traits of cryptogams in Mediterranean ecosystems are driven by water, light and substrate interactions. Journal of Vegetation Science 25: 778-792.

Hauck, M., Bruyn, U, \& Leuschner, C. 2013. Dramatic diversity losses in epiphytic lichens in temperate broad-leaved forests during the last 150 years. Biological Conservation 157: 136-145.

Hammer, O.; Harper, D.A.T. \& Ryan, P.D. 2001. PAST: Paleontological Statistics Software Package for Education and Data Analysis. Disponível em:http://palaeo-electronica.org/2001_1/past/issue1_01. htm Acessado em 12.04.2016.

Instituto Brasileiro de Geografia e Estatística. IBGE. 2016. Disponível em: $<$ http://www.cidades.ibge.gov.br> Acessado em 24.05.2016.

Käffer, M.I., Alves, C., Caceres, M.E.S., Martins, S.M.A. \& Vargas V.M.F. 2011. Caracterização da comunidade liquênica corticícola de Porto Alegre e áreas adjacentes RS, Brasil. Acta Botanica Brasilica 25(4): 832-844.
Käffer, M.I., Dantas, R.V. \& Martins, S.M. de A. 2016a. Characterization of the epiphytic lichen vegetation in a riparian forest in southern Brazil. Plant Ecology and Evolution 149(1): 92-100.

Käffer, M.I., Ganade, G. \& Marcelli, M.P. 2009. Lichen diversity and composition in Araucaria forest and tree monocultures in southern Brazil. Biodiversity and Conservation 18: 3543-3561.

Käffer, M.I., Koch, N.M., Martins, S.M. de A. \& Vargas, V. 2016b. Lichen community versus host tree bark texture in an urban environment in southern Brazil. Iheringia. Série Botânica 71(1): 49-54.

Käffer, M.I., Lemos, A.T., Apel, M.A., Rocha, J.V., Martins, S.M.de A. \& Vargas, V. M.F. 2012. Use of bioindicators to evaluate air quality and genotoxic compounds in an urban environment in Southern Brazil. Environmental Pollution 163: 24-31.

Käffer, M.I., Marcelli, M.P. \& Ganade, G. 2010. Distribution and composition of the lichenized mycota in a landscape mosaic of southern Brazil. Acta Botanica Brasilica 24: 790-802.

Käffer, M.I. \& Martins, S.M.A. 2014. Evaluation of the environmental quality of a protected riparian forest in Southern Brazil. Bosque 35: 325-336.

Käffer, M.I., Martins, S.M.A., Dantas, R.V. \& Maciel, F.C. 2015. Composição da comunidade liquênica em floresta ribeirinha na APA do Ibirapuitã, RS, Brasil. Hoehnea 42(2): 273-288.

Käffer, M.I. \& Martins-Mazzitelli, S.M.A. 2005. Fungos liquenizados corticícolas e terrícolas da área da sub bacia do Sinos e Taquari-Antas, RS, Brasil. Acta Botanica Brasilica 19(4): 813-817.

Kashiwadani, H. \& Kalb, K. 1993. The genus Ramalina in Brazil. Lichenologist 25(1): 1-31.

Koch, N.M., Maluf, R.W. \& Martins, S.M. de A. 2012. Comunidade de liquens foliosos em Piptocarpha angustifolia Dusén ex Malme (Asteraceae) em área de Floresta Ombrófila Mista no estado do Rio Grande do Sul, Brasil. Iheringia. Série Botânica 67(1): 47-57.

Koch, N.M., Martins, S.M. de A., Lucheta, F. \& Müller, S.C. 2013. Functional diversity and traits assembly patterns of lichens as indicators of successional stages in a tropical rainforest. Ecological Indicators 34: 22-30.

Le Blanc, F.S.C. \& de Sloover, J. 1970. Relation industrialization and the distribution and growth of epiphytic lichens and mosses in Montreal. Canadian Journal of Botany 48: 1485-1496.

Lie, M.H., Arup, U., Grytnes, J.A. \& Ohlson, M. 2009. The importance of host tree age, size and growth rate as determinants of epiphytic lichen diversity in boreal spruce forests. Biodiversity and Conservation 18: 3579-3596.

Liira, J.T. \& Sepp, T. 2009. Indicators of structural and habitat natural quality in boreo-nemoral forests along the management gradiente. Annals Botanny Fennici 46: 308-325.

Lucheta, F. \& Martins, S.M.A. 2014. Liquens foliosos e fruticosos corticícolas do Jardim Botânico de Porto Alegre, RS, Brasil. Iheringia. Série Botânica 69(1): 29-35.

Lücking, R., Hodkinson, B.P. \& Leavitt, S.D. 2017. The 2016 classification of lichenized fungi in the Ascomycota and Basidiomycota-Approaching one thousand genera. The Bryologist 119(4): 361-416.

Lücking, R., Rivas-Plata, E., Chaves, J.L., Umaña, L. \& Sipman, H.J.M. 2009. How many tropical lichens are there really?. Bibliotheca Lichenologica 100: 399-418.

Maccheroni, W.J., Araújo W.L. \& Lima, A.O.S. 2010. Ecologia: habitat e interações fúngicas com plantas, animais, fungos e bactérias. In Fungos: uma introdução à biologia, bioquímica e biotecnologia. (E. Esposito \& J.L. Azevedo, eds.). Editora da Universidade de Caxias do Sul, Caxias do Sul, p. 451-476.

Marcelli, M.P. 1992. Ecologia liquênica nos manguezais do sul-sudeste brasileiro. Bibliotheca Lichenologica 47(1): 1-310.

Marcelli, M.P. 1996. Biodiversity assessment in lichenized fungi: the necessary naive roll makers. In A first approach (C.E.M. Bicudo \& N.A. Menezes, eds.). CNPq, São Paulo, p. 93-107.

Marcelli, M.P. 1998. Hystory and current Knowledge of Brazilian Lichenology. In Lichenology in Latin America: history, current knowledge and applications. (M.P. Marcelli \& M.R.D. Seaward, eds.). Cetesb, São Paulo. p. 25-45.

Marcelli, M.P. 2004. Checklist of lichens and lichenicolous fungi of Brazil. Versão 1. Disponível em: <http://www.biologie.unihamburg. de/checklists/brazil 1.htm.> Acessado em 03.11.2015. 
Martins-Mazzitelli, S.M.A., Mota-Filho, F. de O., Pereira, E.C. \& Figueira, R. 2006. In Biologia de Liquens (L.M. Filho, M.E. Legaz, C.V. Cordoba \& E.C. Pereira, eds.). Âmbito Cultural, Rio de Janeiro, p. 101-133.

Martins, S.M.A. \& Marcelli, M.P. 2011. Specific distribution of lichens on Dodonea viscosa L. in the restinga area of Itapuã State Park in Southern Brazil. Hoehnea 38(3): 397-411.

Matos, P., Pinho, P., Aragón, G., Martínez, I., Nunes, A., Soares, A.M.V.M. \& Branquinho, C. 2015. Lichen traits responding to aridity. Journal of Ecology 103: 451-458.

Nash III, T.H. 2008. Introduction. In Lichen Biology (T.H. Nash III, ed.). University Press, Cambridge, p. 1-8.

Novo Hamburgo. 2015. Prefeitura Municipal de Novo Hamburgo. Disponível em: < http://www.novohamburgo.rs.gov.br/> Acessado em 24.11.2015.

Peel, M.C., Finlayson, B.L. \& McMahon, T.A. 2007. Updated world map of the Köppen-Geiger climate classification. Hydrology and Earth System Sciences 11: 1633-1644.

Seaward, M.R.D. 2008. Environmental role of lichens. In Lichen Biology (Nash III, T.H, ed.). Cambridge University Press, Cambridge, p. 274-298.

Sierk, H.A. 1964. The genus Leptogium in North America and north of Mexico. Bryologist 67: 1-317.

Sítio São Luiz. 2015. Disponível em: <http://www.sitiosaoluiz.com.br/>. Acessado em 29.10.2015.
Swinscow, T.D.V. \& Krog, H. 1998. Macrolichens of East Africa. British Museum Natural History. London. 390 p.

Spielmann, A.A. 2006. Check list of lichens and lichenicolous fungi of Rio Grande do Sul (Brazil). Caderno de Pesquisa. Série Biologia 18(2): 7-25.

Spielmann, A.A. \& Marcelli, M.P. 2008. Parmeliaceae (Ascomycota liquenizados) nos barrancos e peraus da encosta da Serra Geral, Vale do rio Pardo, Rio Grande do Sul, Brasil. I. Introdução e chave para gêneros. Iheringia. Série Botânica 63: 159-169.

Spribille, T., Tuovinen, V., Resl, P., Vanderpool, D., Wolinski, H., Aime, C.M., Schneider, K., Stabentheiner, E., Toome-Heller, M., Thor, G., Mayrhofer, H., Johannesson, H. \& McCutcheon, J.P. 2016. Basidiomycete yeasts in the cortex of ascomycete macrolichens. Science 353 (6298): 488-492.

Teixeira, M.B., Coura Neto, A.B., Pastore, U. \& Rangel Filho, A.L.R. 1986. Vegetação. In Levantamento de recursos naturais (IBGE, ed.). Rio de Janeiro, v. 33, p. 541-620.

Universidade Feevale. 2015. Disponível em: http://www.feevale.br/ institucional/como-chegar-a-feevale. Acessado em: 24.04.2015.

Wolseley, P.A. 1991. Observations on the composition and distribution of the 'Lobarion' in forest of southeast Asia. In Tropical lichens: their systematic. (D.J Galloway, ed). Clarendon Press, Oxford, p. 217-243. 